University of Nebraska - Lincoln

DigitalCommons@University of Nebraska - Lincoln

3-8-2005

\title{
Perpendicular exchange bias and its control by magnetic, stress and electric fields
}

Christian Binek

University of Nebraska-Lincoln, cbinek@unl.edu

Pavel Borisov

Universität Duisburg-Essen, p.borisov@lboro.ac.uk

Xi Chen

Angewandte Physik, Universität Duisburg-Essen, Duisburg, Germany

S. Sahoo

University of Nebraska-Lincoln, sarbeswar@gmail.com

Wolfman Kleemann

Angewandte Physik, Universität Duisburg-Essen, Duisburg, Germany, wolfgang.kleemann@uni-due.de

Follow this and additional works at: https://digitalcommons.unl.edu/physicsbinek

Part of the Physics Commons

Binek, Christian; Borisov, Pavel; Chen, Xi; Sahoo, S.; and Kleemann, Wolfman, "Perpendicular exchange bias and its control by magnetic, stress and electric fields" (2005). Christian Binek Publications. 24. https://digitalcommons.unl.edu/physicsbinek/24

This Article is brought to you for free and open access by the Research Papers in Physics and Astronomy at DigitalCommons@University of Nebraska - Lincoln. It has been accepted for inclusion in Christian Binek Publications by an authorized administrator of DigitalCommons@University of Nebraska - Lincoln. 


\title{
Perpendicular exchange bias and its control by magnetic, stress and electric fields
}

\author{
Ch. Binek ${ }^{1}$, P. Borisov ${ }^{2}$, Xi Chen ${ }^{2}$, A. Hochstrat ${ }^{2}$, S. Sahoo ${ }^{1,2}$, and W. Kleemann²,a \\ 1 Department of Physics and Astronomy and the Center for Materials Research and Analysis, 203 Ferguson Hall, \\ University of Nebraska, Lincoln, NE 68588-0111, USA \\ 2 Angewandte Physik, Universität Duisburg-Essen, 47048 Duisburg, Germany
}

Received 15 September 2004

Published online 8 March 2005 - (c) EDP Sciences, Società Italiana di Fisica, Springer-Verlag 2005

\begin{abstract}
Perpendicular exchange bias (PEB) involving perpendicular magnetic anisotropy (PMA) in both the antiferromagnetic (AF) pinning and the ferromagnetic (FM) sensor layer is expected to become important in future perpendicular recording and sensing devices. Further, because of the reduced spin dimensionality, PEB promises to be easier understandable than the conventional planar exchange bias (EB). In addition to its first realization using the Ising-type $\mathrm{AF}$ compounds $\mathrm{FeF}_{2}$ and $\mathrm{FeCl}_{2}$ we have tested control strategies of EB being alternative to the conventional magnetic and thermal ones. Indeed, specific symmetry properties of the pinning layer have been shown to enable mechanical (viz. piezomagnetic via $\mathrm{FeF}_{2}$ ) and electric control (viz. magneto-electric via $\mathrm{Cr}_{2} \mathrm{O}_{3}$ ) of EB, respectively. Electric control promises to become relevant for TMR devices in MRAM technology.
\end{abstract}

PACS. 75.50.Ee Antiferromagnetics - 75.70.Cn Magnetic properties of interfaces (multilayers, superlattices, heterostructures) - 75.70.Kw Domain structure (including magnetic bubbles) $-75.80 .+\mathrm{q}$ Magnetomechanical and magnetoelectric effects, magnetostriction

\section{Introduction}

The phenomenon of exchange bias (EB), the solid shift of a ferromagnetic hysteresis loop along the field axis by a value $H_{E}$ under the exchange interaction with an adjacent antiferromagnetic (AF) cap layer, has been discovered five decades ago by Meiklejohn and Bean $[1,2]$. However, it required the technology of well-defined magnetic thin film fabrication and the relevance of magnetic multilayer in spin-electronics for it to become one of the most significant topics in modern magnetism [3,4]. Extensive research to gain a detailed understanding of the exchange bias phenomenon is still underway [5-7]. Although, it remains doubtful whether a unique mechanism exists, which describes all aspects of the various experimental findings $[3,7]$, it is widely accepted that a net interface magnetization of the antiferromagnet is necessary in order to observe the EB effect. It is the primary task of microscopic EB theories to explain the origin of the AF interface magnetization and its evolution with temperature, magnetic field, and other parameters.

Perpendicular Exchange Bias (PEB) involving perpendicular magnetic anisotropy (PMA) in both the AF pinning and the ferromagnetic (FM) sensor layer is expected to have particular potential in future perpendicular recording and sensing devices [8]. Further, because of the reduced spin dimensionality, $\mathrm{PEB}$ promises to be easier

\footnotetext{
a e-mail: kleemann@uni-duisburg.de
}

understandable than the conventional planar EB, which typically suffers from complex spin arrangements at the interface. The variety of spin structures is accompanied by a multitude of different microscopic mechanisms, which have been suggested in order to explain the peculiarities of the various manifestations of the EB phenomenon [3].

However, apart from the various attempts to model the EB effect on a microscopic level, the simple MeiklejohnBean (MB) expression $\mu_{0} H_{E}=-J S_{\mathrm{AF}} S_{\mathrm{FM}} /\left(M_{\mathrm{FM}} t_{\mathrm{FM}}\right)$ describes the dependence of the bias field $\mu_{0} H_{E}$ on a phenomenological coupling $J$ between the FM and AF interface magnetizations $S_{\mathrm{FM}}$ and $S_{\mathrm{AF}}$, respectively, while $t_{\mathrm{FM}}$ and $M_{\mathrm{FM}}$ are the thickness and the saturation magnetization of the FM layer. The MB formula expresses the fact that additional Zeeman-energy is required to overcome the exchange coupling between $S_{\mathrm{AF}}$ and $S_{\mathrm{FM}}$ at the interface. This simple description can be generalized in order to take into account, e.g., finite anisotropy and thickness of the AF layer [9]. Recently, the dependence on the former parameter has been verified by Lund et al. [10]. This more sophisticated MB-approach predicts also an angular dependence of the exchange bias field on the relative orientation, $\theta$, between the easy axis and the applied magnetic field which goes beyond the simple $\cos \theta$-dependence involved in the scalar product $\underline{S}_{\mathrm{AF}} \cdot \underline{S}_{\mathrm{FM}}$. Nevertheless, a more realistic description of the $\mu_{0} H_{E}$ vs. $\theta$ requires knowledge about details of the magnetization reversal process in the vicinity of both coercive fields, $H_{c 1, c 2}$. Magnetization reversal usually deviates significantly from coherent 


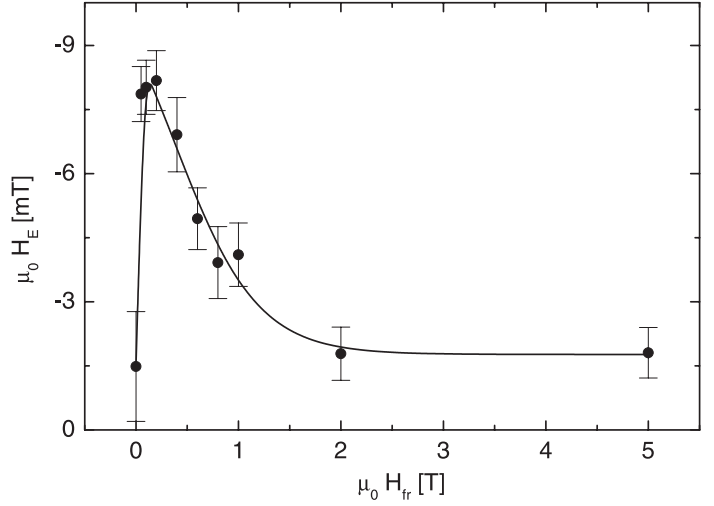

Fig. 1. Dependence of the perpendicular exchange bias in $\mathrm{FeF}_{2}(001) /(\mathrm{Co} / \mathrm{Pt})_{3} / \mathrm{Pt}$ on the freezing field. Experimental data (circles with error bars) and their best fit to a mean-field theory [12] involving Zeeman and exchange energy contributions of Ising-spins in an axial freezing field (solid line) are shown.

rotation at least for one branch of the magnetic hysteresis loop and, hence, simulative approaches are required [11].

It is important to stress, that the phenomenological MB approach does not compete with theories involving microscopic details of the exchange bias mechanism in general and of the AF interface magnetization in particular. The MB expression provides no information about the origin of $S_{\mathrm{AF}}$ and the strength of the global coupling constant $J$. The overestimation of the EB field on the basis of the MB-formula, for instance, originates to a certain extent from the assumption that the phenomenological coupling constant $J$ is related to microscopic exchange interaction constants. They have been naively identified with typical bulk properties of the AF and FM constituents. This latter assumption and the underlying oversimplified microscopic picture cannot be concluded from the MB ansatz. Note, however, that non-trivial conclusions can be drawn from the MB approach. The MB formula does suggest, for instance, that an extrinsic control of the EB field can be achieved by modifying the value of $S_{\mathrm{AF}}$. Here, we report on our recent attempts to modify $S_{\mathrm{AF}}$ by various means.

\section{Perpendicular exchange bias in $\mathrm{FeF}_{2}(001) /(\mathrm{Co} / \mathrm{Pt})_{3}$}

We have first introduced PEB using the (001) surface of single crystalline $\mathrm{FeF}_{2}$ substrate capped by a (111)-textured $(\mathrm{Co} / \mathrm{Pt})_{3}$ multilayer with PMA [12]. Competition between the Zeeman energy and the exchange energy of the spins at the interface controls the value of the EB field when field-cooling (FC) the heterostructure. This mechanism gives rise to a freezing field dependence of the exchange bias field as shown in Figure 1 for the heterosystem $\mathrm{FeF}_{2}(001)(1 \mathrm{~mm}) /\{\mathrm{Co}(0.35 \mathrm{~nm}) / \operatorname{Pt}(1.2 \mathrm{~nm})\}_{3} / \operatorname{Pt}(0.8 \mathrm{~nm})$. The appearing $\mathrm{PEB}$ and its freezing field dependence is realistically described within the framework of a

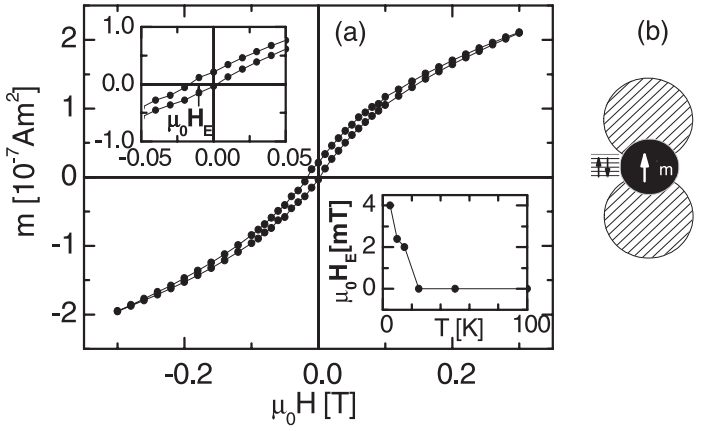

Fig. 2. (a) EB shifted hysteresis curve measured at $T=5 \mathrm{~K}$ in a granular heterolayer $\mathrm{FeCl}_{2} / \mathrm{Fe}$ after $\mathrm{FC}$ with $\mu_{0} H_{\mathrm{fr}}=$ $0.1 \mathrm{~T}$ [19]. The insets show an enlarged plot of the shifted hysteresis loop and the temperature dependence of $H_{E}$, respectively. (b) Schematic sketch of an Fe particle (black circle, FM moment $\underline{m}$ ) and its $\mathrm{FeCl}_{2}$ environment comprising metamagnetically magnetized polar lobes (hatched circles) and exchange coupled equatorial layered AF crystallites (stripes with spin symbols), respectively.

thermodynamic theory involving Ising-type spins underlying both exchange and Zeeman interactions at rough interfaces. Its temperature dependence is successfully mimicked by adding the corresponding behavior of the molecular field [13].

Meanwhile PEB has become a popular subject in magnetic heterolayer research and has been investigated, e.g., on the systems $\mathrm{CoO} /(\mathrm{Co} / \mathrm{Pt})_{4}[14], \mathrm{FeMn} /\left(\mathrm{Co}_{90} \mathrm{Fe}_{10} / \mathrm{Pt}\right)_{4}$ [15], $\mathrm{FeMn} /(\mathrm{Co} / \mathrm{Pt})_{4}[8], \mathrm{FeMn} /(\mathrm{Co} / \mathrm{Pd})_{n}[16]$, and $\mathrm{NiO} /(\mathrm{Co} / \mathrm{Pt})_{3}[17]$.

\section{Exchange bias in $\mathrm{FeCl}_{2} / \mathrm{FM}$ systems}

Another candidate for $\mathrm{PEB}$ is the layered $\mathrm{AF} \mathrm{FeCl}_{2}$, which differs, however, crucially from $\mathrm{FeF}_{2}$ by its pronounced metamagnetic properties [4]. This has drastic consequences onto its applicability in EB heterostructures, where usually the AF partner is assumed to be rather insensitive against the strong FM partner. Hence, as a rule, it is the AF which controls the FM hysteresis. Only minute, albeit essential formation of AF domains during $\mathrm{FC}$ through $T_{\mathrm{N}}$ is involved. This is just the opposite for $\mathrm{FeCl}_{2}(111)$ when being confronted with a "strong" $\mathrm{FM}$ like the multilayer $(\mathrm{Co} / \mathrm{Pt})_{n}$. Here the FM partner is much stronger than the AF one, which exerts but weak pinning forces onto the FM. Due to strong interface exchange with the FM layers it rather decays into a metastable interfacial multidomain state after FC to below $T_{\mathrm{N}}\left(\mathrm{FeCl}_{2}\right) \approx 24 \mathrm{~K}[18]$.

Recently [19] we have evidenced that the magnetic stray fields of single domain nanoparticles of Fe embedded in a thin film of $\mathrm{FeCl}_{2}$ may even give rise to a metamagnetic transformation of the AF environment thus creating "giant" moments at $T<T_{\mathrm{N}}$. After FC only weak EB, $\mu_{0} H_{E} \approx-4 \mathrm{mT}$, is observed and attributed to exchange between the Fe nanoparticles at their equatorial belts with properly orientated $\mathrm{FeCl}_{2}$ environment (Fig. 2). Owing to 


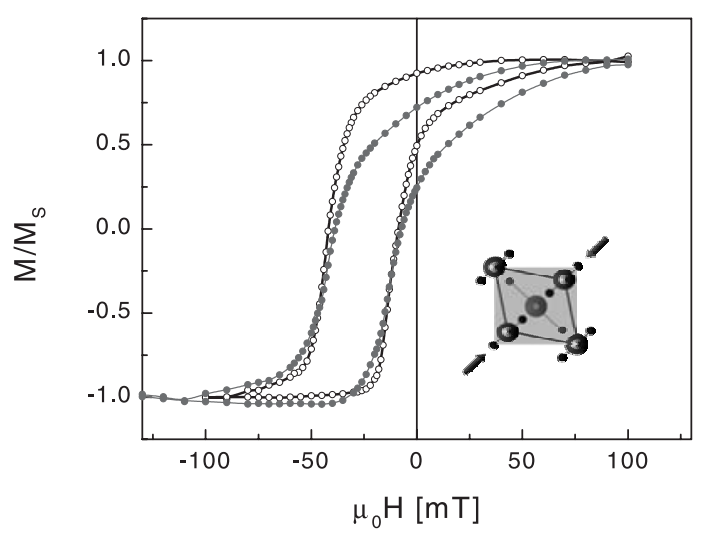

Fig. 3. Magnetic hysteresis after saturation of the Fe layer in $\mathrm{Fe}_{0.6} \mathrm{Zn}_{0.4} \mathrm{~F}_{2}(110) / \mathrm{Fe} / \mathrm{Ag}$ at $T=100 \mathrm{~K}$ and $\mathrm{FC}$ with $\mu_{0} H_{\mathrm{fr}}=$ $5 \mathrm{mT}$ applied along the AF $c$-axis [21]. Open circles indicate the hysteresis under natural shear-stress, while the solid circles denote the hysteresis under external shear-stress $\sigma_{x y}>0$. The inset shows the influence of $\sigma_{x y}>0$ on the ions in a unit cell of $\mathrm{FeF}_{2}$.

the planar configuration of the equatorial Fe spins also the EB is necessarily planar (Fig. 2).

\section{Piezomagnetic control of exchange bias in $\mathrm{FeF}_{2} / \mathrm{Fe}$}

Our first unconventional EB mechanism profits from the piezomagnetic nature of the rutile-type structure of $\mathrm{FeF}_{2}[20]$. Under symmetry-breaking shear stress, different spin-orbit controlled $g$-factors characterize both sublattices. They give rise to a global excess magnetic moment, part of which controls the interface. Experiments were carried out on the layered system $\mathrm{Fe}_{0.6} \mathrm{Zn}_{0.4} \mathrm{~F}_{2}(110)(2 \mathrm{~mm}) / \mathrm{Fe}(14 \mathrm{~nm}) / \mathrm{Ag}(35 \mathrm{~nm})$, where a piezomagnetic moment is created by planar shear stress exerted onto the substrate crystal [21]. Note that an $\mathrm{AF}$ single domain with unique direction of the $\mathrm{AF}$ vector $\underline{l}$ maximizes the moment, while statistical multidomain structures at the AF/FM interface tend to annihilate the piezomagnetic EB.

Figure 3 shows an experimental attempt to evidence that a fraction of the induced piezomagnetic moment contributes to the AF interface magnetization and, hence, to the EB-field. External shear stress $\sigma_{x y}>0$ is applied along the [110] direction of the antiferromagnet. The external shear stress modifies the piezomagnetic moment $m_{z}^{p}=\lambda \sigma_{x y} l_{z} /|\underline{l}|$ when changing the natural stress distribution $\sigma_{x y}(\underline{r})$, where $l_{z}$ is the component of the AF order parameter in [001] direction, and $\lambda$ is a proportionality constant. Figure 3 shows the magnetic hysteresis of $\mathrm{Fe}_{0.6} \mathrm{Zn}_{0.4} \mathrm{~F}_{2}(110) / \mathrm{Fe} / \mathrm{Ag}$ after cooling the heterostructure from $T=100 \mathrm{~K}$ to $10 \mathrm{~K}$ in a freezing field of $\mu_{0} H_{\mathrm{fr}}=5 \mathrm{mT}$ with (squares) and without (circles) external shear stress $\sigma_{x y}>0$. The reduced shift of the hysteresis under external positive shear stress indicates that

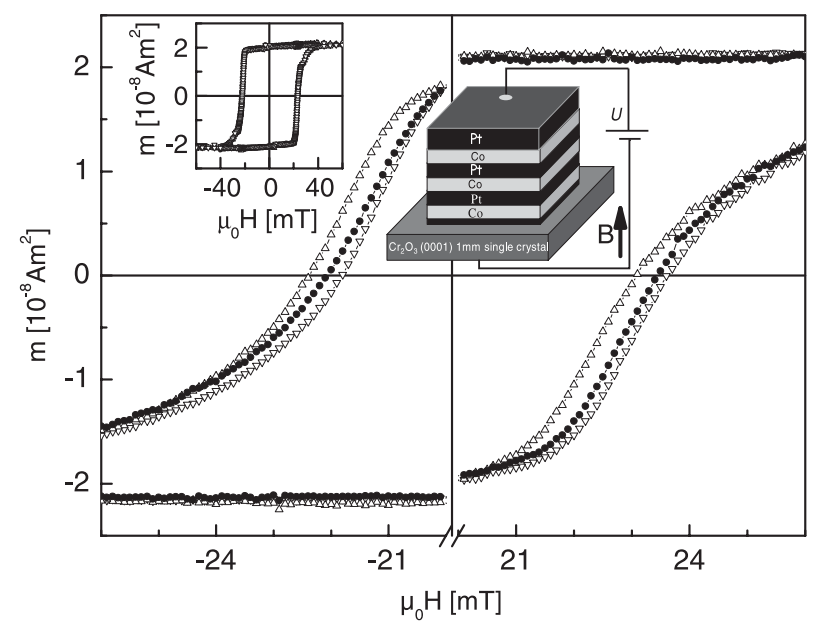

Fig. 4. Magnified hysteresis curves of $\mathrm{Cr}_{2} \mathrm{O}_{3} /(\mathrm{Co} / \mathrm{Pt})_{3}$ measured at $T=150 \mathrm{~K}$ after cooling from $350 \mathrm{~K}$ to $150 \mathrm{~K}$ with both $\mu_{0} H_{\mathrm{fr}}=0.5 \mathrm{~T}$ and $E_{\mathrm{fr}}=-460 \mathrm{kV} / \mathrm{m}$ (up triangles), 0 (solid circles) and $425 \mathrm{kV} / \mathrm{m}$ (down triangles), respectively. The insets show a global view of the same hysteresis curves in the field range $\left|\mu_{0} H\right| \leq 60 \mathrm{mT}$ and a schematic view of the sample as exposed to the external magnetic (B) and electric field (voltage $U$ ).

the built-in stress distribution has a negative spatial average. The external stress, which has not been quantified in the framework of this experiment, changes the EB field substantially, from $25.3 \mathrm{mT}$ to $23.1 \mathrm{mT}$.

\section{Electric control of exchange bias in $\mathrm{Cr}_{2} \mathrm{O}_{3} /(\mathrm{Co} / \mathrm{Pt})_{3}$}

A second unconventional EB mechanism resides on the appearance of a magnetic moment under the action of an external electric field [22]. This ultimate possibility is offered by magneto-electric (ME) [23] compounds, the most prominent (and first [24]) example found in nature is the rhombohedral $\mathrm{AF} \mathrm{Cr}_{2} \mathrm{O}_{3}$. Its corundum structure loses time reversal $(\Theta)$ and inversion symmetry $(P)$ below $T_{\mathrm{N}}=308 \mathrm{~K}$, where it satisfies the operation $\Theta P$ instead [23]. The ME response is macroscopically characterized by the ME susceptibility tensor $\underline{\alpha}$. In the case of $\mathrm{Cr}_{2} \mathrm{O}_{3}$, this tensor of rank two has a simple diagonal structure, where $\alpha_{x x}=\alpha_{y y}=\alpha_{\perp}$ and $\alpha_{z z}=\alpha_{\|}$. In the simplest microscopic description, $\mathrm{Cr}^{3+}$ ions located on different sublattices are shifted by the electric field to nonequivalent positions and experience different strengths of the crystal field. This changes the $g$-tensor and the single ion anisotropy in a non-equivalent way and modifies the exchange integrals between the ions. Largest ME EB effects are intuitively expected for AF single domains, which are conveniently achieved by so-called ME annealing, viz. by cooling to below $T_{\mathrm{N}}$ under the simultaneous action an axial magnetic and electric freezing field, $H_{\mathrm{fr}}$ and $E_{\mathrm{fr}}$, respectively [25]. Both of them are applied perpendicularly to the layer system as shown in the inset to Figure 4. 

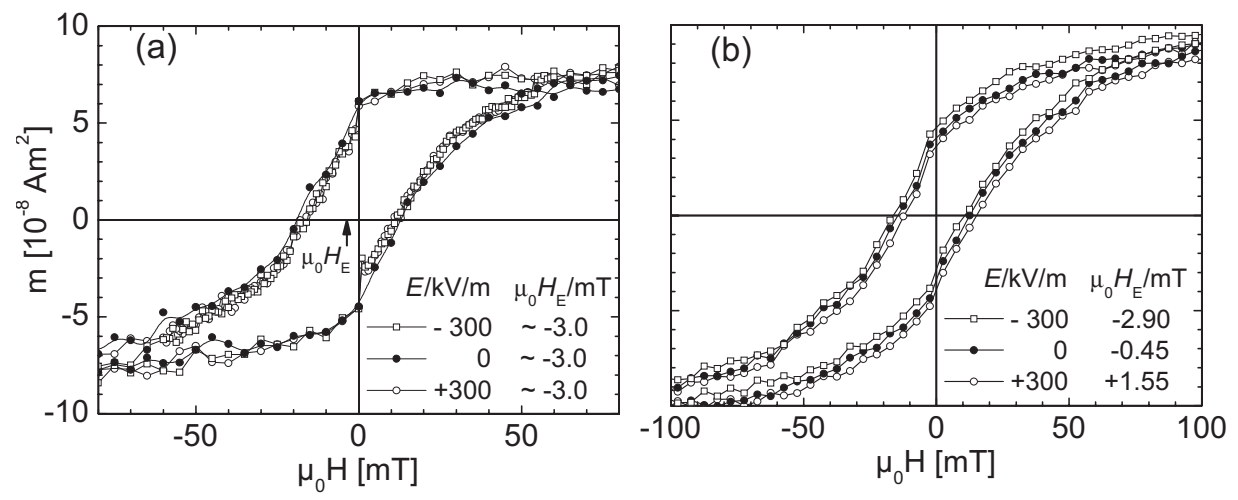

Fig. 5. Hysteresis curves of $\mathrm{Cr}_{2} \mathrm{O}_{3} /(\mathrm{Co} / \mathrm{Pt})_{3}$ measured at $T=150 \mathrm{~K}$ after ME freezing with $\mu_{0} H_{\text {fr }}=0.5 \mathrm{~T}$ and $E_{\text {fr }}=0(\mathrm{a})$ and $\mu_{0} H_{\mathrm{fr}}=0.5 \mathrm{~T}$ and $E_{\mathrm{fr}}=300 \mathrm{kV} / \mathrm{m}$ (b), respectively, under applied axial fields $E=0$ and $E= \pm 300 \mathrm{kV} / \mathrm{m}$, respectively.

The main panel shows that this procedure already changes the conventional EB as measured in zero external electric field on the heterosystem $\mathrm{Cr}_{2} \mathrm{O}_{3}(111)(1 \mathrm{~mm}) / \mathrm{Pt}(0.7 \mathrm{~nm}) /$ $(\mathrm{Co}(0.3 \mathrm{~nm}) / \operatorname{Pt}(1.2 \mathrm{~nm}))_{3}$. After freezing from $350 \mathrm{~K}$ to $150 \mathrm{~K}$ under both $\mu_{0} H_{\mathrm{fr}}=0.5 \mathrm{~T}$ and $E_{\mathrm{fr}}=-460 \mathrm{kV} / \mathrm{m}$ (up triangles), 0 (solid circles) and $425 \mathrm{kV} / \mathrm{m}$ (down triangles), respectively, three mutually shifted nearly rectangular (see inset) hysteresis curves are obtained. As $E_{\mathrm{fr}}$ increases $\mu_{0} H_{E}$ monotonically increases from $0.35 \mathrm{mT}$ via $0.8 \mathrm{mT}$ to $0.9 \mathrm{mT}$, respectively. First of all, the positive sign of the EB seems to indicate weak AF interface coupling, $J<0$, while aligning the coupled $\mathrm{AF}$ and FM moments parallel upon cooling in quite moderate fields [12]. Second, the size of the EB obviously depends on the AF domain state, which is expected to be single domain, $A$ and $B$, say, when applying $E_{\text {fr }} \neq 0$, but will be multidomain, " $A+B$ ", in the case $E_{\mathrm{fr}}=0$. The latter is due to the combined effect of the homogeneous magnetic field and the roughness of the AF/FM interface [5]. Obviously we encounter constructive or destructive effects due to differently registered AF domains, which are formed during ME annealing in the bulk of the AF crystal. By symmetry, positive or negative bulk magnetic moments do arise, which couple to the interface and probably enhance or decrease $S_{\mathrm{AF}}$, respectively. After removing $E_{\mathrm{fr}}$ net positive or negative contributions to $S_{\mathrm{AF}}$ with respect to the case of an AF multidomain under $E_{z}=0$ will survive and shift the loops as shown in Figure 4.

Similar results are shown in Figure 5 on another sample of $\mathrm{Cr}_{2} \mathrm{O}_{3}(111) /(\mathrm{Co} / \mathrm{Pt})_{3}$, which was prepared under $\mu_{0} H_{\mathrm{fr}}=0.5 \mathrm{~T}$ and $E_{\mathrm{fr}}=0$ (a) and $\mu_{0} H_{\mathrm{fr}}=0.5 \mathrm{~T}$ and $E_{\mathrm{fr}}=300 \mathrm{kV} / \mathrm{m}$ (b). Let us first consider the exchange bias of the hysteresis loops when measured in zero external electric field. In agreement with the above experience (Fig. 4) we observe a positive shift of $\mu_{0} H_{E}$ from $-3 \mathrm{mT}$ (a) to $-0.45 \mathrm{mT}$ (b). The negative EB field values hint at slightly stronger (AF) interface coupling in this particular sample. Then the magneto-electric effect is tested by taking the same loops again, but under the action of an external electric field, $E= \pm 300 \mathrm{kV} / \mathrm{m}$. Interestingly, the merely magnetically cooled sample (a) does not show any significant shift of the loop. This is understandable, since the ME excess interface moments have either sign in the multidomain sample thus prepared and do not change the as-frozen value of $S_{\mathrm{AF}}$. This changes drastically for the ME cooled sample (b), where the external field $E= \pm 300 \mathrm{kV} / \mathrm{m}$ unambiguously shifts the loop by $\delta\left(\mu_{0} H_{E}\right) \approx \pm 2 \mathrm{mT}$ as expected for the ME controlled EB.

\section{Outlook}

Although the ME induced EB effect is quite small (Fig. 5), [22], this drawback will certainly be overcome when using oriented thin films of $\mathrm{Cr}_{2} \mathrm{O}_{3}(111)$ and more suitable coupling layers to the $(\mathrm{Co} / \mathrm{Pt})_{\mathrm{n}}$ multilayer stack. Thin films will easily allow to establish very high electric fields with low voltages. This is a prerequisite for applications of ME thin films. One of them might be their use as dielectric tunnel junctions between two FM layers. They may thus be implemented as electric controlled pinning layers in spin valve structures. This promises to have a significant impact on future spintronic devices. On the one hand it opens an alternative path to current induced switching and on the other hand it provides a novel electric field control of magnetic states in modern devices involving multiferroic systems.

Thanks are due to DFG within the framework of SFB 491 "Magnetic heterolayers - structure and electronic transport" for financial support.

\section{References}

1. W.H. Meiklejohn, C.P. Bean, Phys. Rev. Lett. 102, 1413 (1956)

2. W.H. Meiklejohn, C.P. Bean, Phys. Rev. 105, 904 (1957)

3. J. Nogués, I.K. Schuller, J. Magn. Magn. Mater. 192, 203 (1999)

4. Ch. Binek, Ising-type Antiferromagnets: Model Systems in Statistical Physics and in the Magnetism of Exchange Bias, Springer Tracts Mod. Phys., Vol. 196 (Springer-Verlag, Berlin, Heidelberg, 2003) 
5. A.P. Malozemoff, Phys. Rev. B 35, 3679 (1987)

6. R.L. Stamps, J. Phys. D: Appl. Phys. 33, R247 (2000)

7. An instructive summary of open questions in the field of exchange bias can be found at http://ischuller.ucsd.edu/EBManifesto.pdf

8. F. Garcia, F. Fettar, S. Auffret, B. Rodmacq, B. Dieny, J. Appl. Phys. 93, 8397 (2003)

9. Ch. Binek, A. Hochstrat, W. Kleemann, J. Magn. Magn. Mater. 234, 353 (2001)

10. M.S. Lund et al., Phys. Rev. B 66, 054422 (2002)

11. B. Beckmann, U. Nowak, K.D. Usadel, Phys. Rev. Lett. 91, 187201 (2003)

12. B. Kagerer, Ch. Binek, W. Kleemann, J. Magn. Magn. Mater. 217, 139 (2000)

13. Ch. Binek, B. Kagerer, S. Kainz, W. Kleemann, J. Magn. Magn. Mater. 226-230, 1814 (2001)

14. S. Maat, K. Takano, S.S.P. Parkin, E.E. Fullerton, Phys. Rev. Lett. 87, 087202 (2001)
15. F. Garcia, G. Casall, S. Auffret, B. Rodmacq, B. Dieny, J. Appl. Phys. 91, 6905 (2002)

16. C.H. Marrows, Phys. Rev. B 68, 012405 (2003)

17. Z.Y. Liu, J. Magn. Magn. Mater. 281, 247 (2004)

18. Ch. Binek, A. Hochstrat, W. Kleemann, Phys. Stat. Sol. (a) 189, 575 (2001)

19. S. Sahoo, Ch. Binek, W. Kleemann, Phys. Rev. B 68, 174431 (2003)

20. A.S. Borovik-Romanov, Sov. Phys. JETP 11, 786 (1960)

21. Ch. Binek, Xi Chen, A. Hochstrat, W. Kleemann, J. Magn. Magn. Mater. 240, 257 (2002)

22. A. Hochstrat, Ch. Binek, Xi Chen, W. Kleemann, J. Magn. Magn. Mater. 272-276, 325 (2004)

23. L.D. Landau, E.M. Lifshitz, Electrodynamics in continuous media (Nauka, Moscow, 1952)

24. D.N. Astrov, Sov. Phys. JETP 11, 780 (1960)

25. T.H. O'Dell, Phil. Mag. 13, 921 (1966) 